\title{
Family Hierarchy and Large Neutrino Mixings
}

\author{
Fu-Sin Ling ${ }^{1}$ and Pierre Ramond ${ }^{2}$ \\ Institute for Fundamental Theory \\ Department of Physics, University of Florida, \\ Gainesville, FL, 32611, USA
}

\begin{abstract}
The recent neutrino data seem to favor two large and one small mixing angles and a hierarchy of their squared mass differences. We discuss these within the context of hierarchical neutrino masses. We show that this scheme suggests a specific neutrino mass matrix with mild fine-tuning. We then present a Froggatt-Nielsen model that reproduces this matrix.
\end{abstract}

\footnotetext{
${ }^{1}$ e-mail: fsling@phys.ufl.edu

${ }^{2}$ e-mail: ramond@phys.ufl.edu
} 


\section{Introduction}

Recently, numerous neutrino experiments have confirmed the first true signal of physics beyond the standard model. The flavor conversion of neutrinos, or neutrino oscillations has been observed in both the atmospheric and the solar neutrinos. Therefore, unlike in the Standard Model, neutrinos do have mass and mixings.

The atmospheric neutrino data are likely explained by a $\nu_{\mu}-\nu_{\tau}$ oscillation with parameters [1, 2]

$$
\begin{aligned}
\Delta m^{2} & \simeq 2.5 \cdot 10^{-3} e V^{2} \\
\sin ^{2} 2 \theta & \geq 0.8
\end{aligned}
$$

For the solar neutrinos deficit, there are four different solutions, called Large Mixing Angle (LMA), Small Mixing Angle (SMA), "Low", and the vacuum oscillation solution (VO). Typical values of the mixing angle and the $\Delta m^{2}$ at best fit are shown in table 13 . These solutions are obtained by

\begin{tabular}{|c|c|c|}
\hline Solution & $\tan ^{2} \theta$ & $\Delta m^{2}\left(\mathrm{eV}^{2}\right)$ \\
\hline LMA & $3 \cdot 10^{-1}$ & $3 \cdot 10^{-5}$ \\
SMA & $10^{-3}$ & $7 \cdot 10^{-6}$ \\
LOW & $6 \cdot 10^{-1}$ & $10^{-7}$ \\
VO & $3 \cdot 10^{-1}$ or 3 & $8 \cdot 10^{-11}$ \\
\hline
\end{tabular}

Table 1: Typical values of $\tan ^{2} \theta$ and $\Delta m^{2}$ for the various solar neutrinos solutions at best fit.

combining the rates observed at different types of experiments (Homestake, Gallex, Sage, SuperKamiokande (SK), SNO). However, in addition, each solution has a characteristic signature in various observables like the energy spectrum, the day-night asymmetry, the seasonal variation, or the zenith angle dependence. Even though a possible distortion is small and difficult to measure, the huge statistics accumulated by SK made it possible to almost exclude the $\mathrm{VO}$ and the SMA solutions 4. Moreover, the first results of the ongoing experiment SNO not only demonstrate the existence of flavor conversion of the solar neutrinos independently of any solar model [5], but also seem to favor the LMA solution as some day-night effect is measured [6]. The third signal of neutrino oscillations from LSND [7] needs confirmation. 
If we are to believe these data, and if we assume a framework with only three flavors $\left(\nu_{e}, \nu_{\mu}, \nu_{\tau}\right)$, then the neutrino sector exhibits two large mixing angles, and one small mixing angle, imposed by the CHOOZ [8] experiment. At first sight, mass hierarchies and mixing patterns are completely different in the lepton and in the quark sectors. For the quarks, the observed mixing matrix is given by the CKM matrix, which is almost the unit matrix. The order of magnitude of the off-diagonal elements are given as a power of the Cabibbo angle $\lambda_{c}$ in the Wolfenstein parametrization. The corresponding MNS matrix in the lepton sector, which is now measured in the neutrino oscillation experiments, does not however seem to fit in such parametrization scheme if two large mixing angles are indeed observed.

In this paper, we analyze the requirements that are needed in the effective neutrino Majorana mass matrix in order to give rise to two large and one small mixing angles, in the context of hierarchical neutrino masses. The derived structure enables us to construct a model of family hierarchy based on additional Abelian family symmetries, in the spirit of [11, 12, which is compatible with the neutrino observations. It turns out that the hierarchies and mixings among leptons and quarks are highly interrelated in this model, in sharp contrast with the picture that appears at first glance.

The paper is organized as follows. In section 2, we analyze the effective $3 \times 3$ neutrino mass matrix. In section 3 , we present a new model of family symmetry and its predictions for the neutrinos, charged leptons and quarks masses and mixings. The internal consistency of the model, including anomaly cancellation is then briefly discussed.

\section{Neutrino mass hierarchies and large mixing angles}

In this section, we derive the structure of the $3 \times 3$ effective neutrino mass matrix assuming a hierarchical pattern of the neutrino masses.

As neutrino experiments are only sensitive to the values of $\Delta m^{2}$, but not to the absolute values of the neutrinos masses, the range of the neutrino masses could in principle be different from the measured splittings. For example, if $m_{\nu} \sim 1 \mathrm{eV}$, which could be useful for cosmology purposes, the observed $\Delta m^{2}$ would result in a hyperfine-like splitting structure in the neutrino sector, totally different from what is observed in the other fermionic 
sectors.

In what follows, we assume that a mass hierarchy takes place in the neutrino sector, and that it is responsible of the observed hierarchy between $\Delta m_{\odot}^{2}$ for solar neutrinos and $\Delta m_{\oplus}^{2}$ for atmospheric neutrinos ${ }^{3}$. For example, for the LMA solution, we have

$$
\frac{\Delta m_{\odot}^{2}}{\Delta m_{\oplus}^{2}} \simeq 10^{-2}
$$

Therefore, we suppose that the neutrinos masses can be written as $\widetilde{M}_{\nu}=$ $m_{0} \cdot \operatorname{diag}\left(\beta \lambda^{b}, \alpha \lambda^{a}, 1\right)$, where $\lambda$ is some small parameter, $b \geq a>0$ are integers and $\alpha, \beta$ are numerical coefficients of order 1 . Then, in the charged leptons mass eigenstates basis, the full effective mass matrix will be

$$
M_{\nu}=U_{M N S} \cdot \widetilde{M}_{\nu} \cdot U_{M N S}^{t}
$$

with

$$
U_{M N S} \simeq\left(\begin{array}{ccc}
\cos \theta_{\odot} & -\sin \theta_{\odot} & \ll 1 \\
\sin \theta_{\odot} \cos \theta_{\oplus} & \cos \theta_{\odot} \cos \theta_{\oplus} & -\sin \theta_{\oplus} \\
\sin \theta_{\odot} \sin \theta_{\oplus} & \cos \theta_{\odot} \sin \theta_{\oplus} & \cos \theta_{\oplus}
\end{array}\right)
$$

The structure found for $M_{\nu}$ is

$$
M_{\nu} \simeq m_{0} \cdot\left(\begin{array}{ccc}
\gamma \lambda^{c} & \delta_{1} \lambda^{d} & \delta_{2} \lambda^{d} \\
\delta_{1} \lambda^{d} & \sin ^{2} \theta_{\oplus} & -\sin \theta_{\oplus} \cos \theta_{\oplus} \\
\delta_{2} \lambda^{d} & -\sin \theta_{\oplus} \cos \theta_{\oplus} & \cos ^{2} \theta_{\oplus}
\end{array}\right)
$$

where $\gamma, \delta_{1}$ and $\delta_{2}$ are some numerical factors. We see that the large value of the atmospheric mixing angle forces all the elements in the 2-3 sub-block of $M_{\nu}$ to be $\mathcal{O}(1)$. Moreover, the hierarchy of the neutrinos masses forces the determinant of the 2-3 sub-block, referred below as the sub-determinant $D_{1}$ to be zero, i.e. $D_{1} \ll 1$. The effect of the second large mixing angle is more subtle to analyze. Basically, it amounts to a relation between $D_{1}$ and the full $3 \times 3$ determinant of coefficients $D_{0}$.

A structure of the neutrino mass matrix similar to the one found in (4) can be derived in models with an Abelian family symmetry. However, the additional condition on the sub-determinant $D_{1}$ is not generic in models of

\footnotetext{
${ }^{3}$ Therefore, we ignore as well the possibility of the so-called inverted hierarchy, where $m_{1} \simeq-m_{2} \gg m_{3}$, and $\Delta m_{\odot}^{2}=m_{1}^{2}-m_{2}^{2}$, which also gives a large atmospheric mixing angle [9]
} 
family hierarchy. Different possible mechanisms to solve this problem have been advocated [10]. In what follows, we also focus on the conditions on $D_{0}$ and $D_{1}$ to get a large solar mixing angle in addition to the large atmospheric angle.

As an illustrative example, we take the prediction for the neutrino mass matrix in the model of ref. [11,

$$
M_{\nu}^{(1)} \sim m_{0}\left(\begin{array}{ccc}
\lambda^{6} & \lambda^{3} & \lambda^{3} \\
\lambda^{3} & 1 & 1 \\
\lambda^{3} & 1 & 1
\end{array}\right)
$$

where $\lambda$ is of the order of the Cabibbo angle $\lambda \simeq \lambda_{c}$. Generically, i.e. without any assumption on the numerical prefactors, the solar mixing angle obtained is small, $\theta_{\odot} \sim \mathcal{O}\left(\lambda^{3}\right)$, and the mass spectrum is $m_{\nu} \sim m_{0} \cdot\left(\lambda^{6}, 1,1\right)$, which does not result in the desired hierarchy of the $\Delta m^{2}$. As noticed in ref. 13, two large mixing angles and the hierarchy of $\Delta m^{2}$ can be obtained, but it requires to fine-tune the sub-determinant to order $\mathcal{O}\left(\lambda^{3}\right)$. More precisely, we can summarize (see table 2) the mixing and hierarchies obtained when $D_{0} \sim \mathcal{O}(1)$ and $D_{1} \sim \mathcal{O}\left(\lambda^{p}\right)$, with $p \geq 0$ integer.

\begin{tabular}{|c|c|c|c|}
\hline$D_{0} \sim \mathcal{O}(1), D_{1} \sim \mathcal{O}\left(\lambda^{p}\right)$ & $m_{\nu}\left(\cdot m_{0}\right)$ & $\theta_{\odot}$ & $\Delta m_{\odot}^{2} / \Delta m_{\oplus}^{2}$ \\
\hline$p=0$ & $\lambda^{6}, 1,1$ & $\lambda^{3}$ & $\mathcal{O}(1)$ \\
$1 \leq p<3$ & $\lambda^{6-p}, \lambda^{p}, 1$ & $\lambda^{3-p}$ & $\lambda^{2 p}$ \\
$p=3$ & $\lambda^{3}, \lambda^{3}, 1$ & $\mathcal{O}(1)$ & $\lambda^{6}$ \\
$3<p<6$ & $\lambda^{3}, \lambda^{3}, 1$ & $\pi / 4$ & $\lambda^{p+3}$ \\
$p \geq 6$ & $\lambda^{3}, \lambda^{3}, 1$ & $\pi / 4$ & $\lambda^{9}$ \\
\hline
\end{tabular}

Table 2: Neutrino mixing and hierarchies from $M_{\nu}^{(1)}$ as a function of $p$.

As we can see, with the mass matrix $M_{\nu}^{(1)}$, two large mixing angles result only if $D_{1}$ is precisely fine-tuned to order $\mathcal{O}\left(\lambda^{3}\right)$. For $p>3$, the solar angle $\theta_{\odot}$ becomes maximal, and this is excluded at $99 \%$ C.L. by the experimental data [3]. Moreover, for $p=3$, the value of the ratio $\Delta m_{\odot}^{2} / \Delta m_{\oplus}^{2}$ would favors the "LOW" solution which only exists at 99\% C.L. if all observed rates are combined and day-night asymmetry is taken into account. Therefore, we conclude that two large mixing angles cannot be reproduced by the matrix $M_{\nu}^{(1)}$ without strong fine-tuning. 
On the other hand, the following neutrino mass matrix, $M_{\nu}^{(2)}$, which has the same structure as $M_{\nu}^{(1)}$, is in good agreement with all the neutrino data without strong fine-tuning.

$$
M_{\nu}^{(2)} \sim m_{0}\left(\begin{array}{ccc}
\lambda^{2} & \lambda & \lambda \\
\lambda & 1 & 1 \\
\lambda & 1 & 1
\end{array}\right)
$$

with again $\lambda \simeq \lambda_{c}$. The CHOOZ constraint is easily satisfied because $U_{e 3} \sim \mathcal{O}(\lambda)^{4}$. When $D_{0} \sim \mathcal{O}(1)$ and $D_{1} \sim \mathcal{O}(\lambda)$ (which is a mild assumption), one gets two large mixing angles and $\Delta m_{\odot}^{2} / \Delta m_{\oplus}^{2} \sim \mathcal{O}\left(\lambda^{2}\right)$. The properties of the mass matrix $M_{\nu}^{(2)}$ have also been investigated using a random coefficients generator [15, 16], and it appears that the probability of a successful phenomenological description of the neutrino data becomes maximal for $\lambda \simeq \lambda_{c}$ [16]. The same structure for the neutrino mass matrix also appears in several different contexts [17, 18, 19.

Moreover, with the matrix $M_{\nu}^{(2)}$, there is a special case which gives rise to two large mixing angles, which may become relevant as the neutrino masses are generated through the seesaw [20] mechanism. We note from table 2 that a large solar mixing angle arises when the two light eigenvalues $\mu_{1}$ and $\mu_{2}$ are of the same order of magnitude.

$$
\mu_{1} \sim \mu_{2} \Longrightarrow \sin \theta_{\odot} \sim \mathcal{O}(1)
$$

The characteristic equation for $M_{\nu}^{(2)}$ is of the form

$$
\mu^{3}+\mathcal{O}(1) \cdot \mu^{2}+\left(D_{1}+\mathcal{O}\left(\lambda^{2}\right)\right) \mu+D_{0} \cdot \lambda^{2}=0
$$

Therefore, the solar mixing angle becomes large when $D_{0} \sim \mathcal{O}\left(\lambda^{2}\right)$ and $D_{1} \sim$ $\mathcal{O}\left(\lambda^{2}\right)$ because we have $\mu_{1} \sim \mu_{2} \sim \mathcal{O}\left(\lambda^{2}\right)$ in this case. The analytic values of the eigenvalues and the mixing matrix can be found in the appendix. The reason why this special case might be relevant is simple. The light neutrino masses are given by a seesaw mechanism. In the chiral Froggatt-Nielsen type models, the charges of the right-handed neutrinos are canceled in the seesaw, and the Cabibbo structure of the effective neutrino mass matrix only depends on the charges of the lepton doublets $L_{i}$ (see [11]). However, the numerical coefficients, or pre-factors are given by the usual see-saw formula

$$
C_{\nu}=-C_{D} \cdot C_{0}^{-1} \cdot C_{D}^{t}
$$

\footnotetext{
${ }^{4}$ In models which generically predict three large mixing angles, like models of neutrino anarchy [14, the ChOOZ constraint becomes critical
} 
where $C_{\nu}, C_{D}$ and $C_{0}$ are the $3 \times 3$ matrices of pre-factors corresponding to the effective light neutrino mass matrix, the Dirac mass matrix and the heavy neutrino Majorana mass matrix respectively. Let's now suppose that the coefficients of the second and of the third lines of $C_{D}$, are approximately (to order $\mathcal{O}(\lambda)$ ) equal, so that the matrix $C_{D}$ can be written as

$$
C_{D}=\left(\begin{array}{ccc}
a & b & c \\
d_{0}+d_{1} \lambda & e_{0}+e_{1} \lambda & f_{0}+f_{1} \lambda \\
d_{0}-d_{1} \lambda & e_{0}-e_{1} \lambda & f_{0}-f_{1} \lambda
\end{array}\right)
$$

where all the coefficients are $\mathcal{O}(1)$. Then, after see-saw, the matrix $C_{\nu}$ indeed verifies $D_{0} \sim \mathcal{O}\left(\lambda^{2}\right)$ and $D_{1} \sim \mathcal{O}\left(\lambda^{2}\right)$. This form for the matrix $C_{D}$ can appear if for example the Lagrangian is symmetric under the exchange of the lepton doublets of the second and the third family, so that we call this symmetry $P_{23}$. If instead the lagrangian is antisymmetric, the corresponding approximate $-P_{23}$ symmetry would have the same consequences. On the other hand, because $\lambda$ is not a very small expansion parameter, the appearance of an approximate $P_{23}$ symmetry in the matrix $C_{D}$ does not require a fundamental symmetry of the lagrangian. We will further address this question in the context of our family symmetry model. If this approximate $P_{23}$ (or $-P_{23}$ ) discrete symmetry is realized, then the pattern of eigenvalues becomes

$$
m_{0} \cdot\left(\lambda^{2}, \lambda^{2}, 1\right)
$$

so that $\Delta m_{\odot}^{2} / \Delta m_{\oplus}^{2} \sim \mathcal{O}\left(\lambda^{4}\right)$. Moreover, the atmospheric mixing angle is automatically maximal $\sin ^{2} 2 \theta_{\oplus} \simeq 1$, as suggested by the experimental data. We emphasize that a maximal atmospheric mixing angle is in general not a feature of models with a family symmetry. Statistically [16, the spread is rather large, so that the experimental bound

$$
\sin ^{2} 2 \theta_{\oplus} \geq 0.8
$$

is in general not automatically satisfied, even if the model predicts a large atmospheric mixing angle.

To conclude this section, we have found that the matrix $M_{\nu}^{(2)}$ with $\lambda \simeq \lambda_{c}$ can accommodate in different ways all the present data on neutrino oscillations (exception made for LSND) with very little fine-tuning. In a context of models with family hierarchies, this structure is suggested by the neutrino oscillation data if the LMA solution to the solar neutrino problem is 
favored, and the mild measured hierarchy between the $\Delta m^{2}$ is reflected in the mild hierarchical pattern of $M_{\nu}^{(2)}$. In the rest of the paper, we present a Froggatt-Nielsen type model which reproduces our favored neutrino mass matrix (6).

\section{A model of family hierarchy}

This model of family hierarchy uses extra Abelian flavor symmetries to reproduce the observed hierarchies among quarks and leptons, in the spirit of an effective theory, as suggested by Froggatt and Nielsen [21]. It is simpler but analogous in its setup to the model in 11, 12. We refer the interested reader to these papers for the general features of the framework, and turn now to the specific points of the model.

\subsection{The family symmetries}

The gauge sector contains the Standard Model groups and two additional Abelian symmetries, $U(1)_{Y_{F}}$ and $U(1)_{X}$. It may also contain some hidden gauge group, so that the complete gauge structure looks like

$$
G_{v i s i b l e} \times U(1)_{Y_{F}} \times U(1)_{X} \times G_{\text {hidden }}
$$

and

$$
G_{\text {visible }} \supseteq S U(3) \times S U(2) \times U(1)_{Y}
$$

The chiral matter content is given by the representation 16 of $S O(10)$. $S O(10)$ contains one $U(1)$ symmetry out of the Standard Model.

$$
S O(10) \supset S U(5) \times U(1)_{V}
$$

The 16 multiplet includes all the fermion fields of the Standard Model, plus a right-handed neutrino which is necessary to implement the see-saw mechanism.

$$
16=10_{1}+\overline{\mathbf{5}}_{-3}+\mathbf{1}_{5}
$$

The symmetry $U(1)_{Y_{F}}$ is traceless over the three families of quarks and leptons and is non-anomalous. Its form is given by

$$
Y_{F}=\eta_{1}(1,0,-1)+\eta_{2}(0,1,-1)
$$


with

$$
\eta_{1}=-\frac{V+7 V^{\prime}}{6}, \quad \eta_{2}=-\frac{V+V^{\prime}}{6}
$$

and $V^{\prime}=1$ for all the matter fields in the $\mathbf{1 6}$ multiplet ( $V^{\prime}$ may be viewed as the extra $U(1)$ in the embedding $\left.E_{6} \supset S O(10) \times U(1)_{V^{\prime}}\right)$. One can notice that the family symmetry $U(1)_{Y_{F}}$ lies outside of $S U(5)$. As a result, all the different fields in the same $S U(5)$ multiplet have the same family structure. Therefore, the hierarchies and mixings predicted in the lepton and in the quark sectors are strongly related in this model.

The symmetry $U(1)_{X}$ is family independent and necessarily anomalous. Its presence is needed in order to construct the right-handed neutrinos Majorana mass matrix. It also participates in the intrafamily hierarchy, namely in the ratio $m_{b} / m_{t}$ of the bottom quark mass to the top quark mass. Over the three chiral families, it has the following form

$$
X=\alpha+\beta V
$$

where $\alpha$ and $\beta$ are coefficients to be determined. The anomalies linear in $X$ in the effective theory are taken care of by the Green-Schwarz anomaly cancellation mechanism [22]. All the other anomalies in the model must vanish.

To construct the quarks and leptons mass matrices, the model also contains two pairs pairs $\left(H_{u}, H_{d}^{\prime}\right)$ and $\left(H_{u}^{\prime}, H_{d}\right)$ in the Higgs sector. For the sake of anomaly cancellation, they are vector-like with respect to the additional Abelian symmetries. Among them, only $H_{u}$ and $H_{d}$ will be coupled to the matter fields to form the supersymmetric and gauge invariants in the superpotential.

Two chiral superfields $\theta_{1}$ and $\theta_{2}$ are needed to mediate the breaking of the flavor symmetries. We express their charges in the matrix $A$

$$
A=\left(\begin{array}{cc}
X\left(\theta_{1}\right) & X\left(\theta_{2}\right) \\
Y_{F}\left(\theta_{1}\right) & Y_{F}\left(\theta_{2}\right)
\end{array}\right)
$$

These charges are constrained by the vacuum structure, and the phenomenological requirement that the coupling of the fields $\theta$ to the Standard Model invariants reproduce the observed hierarchies. These constraints are sufficient to fix the matrix $A$ since all the elements of $A$ and $A^{-1}$ must be integer. We have

$$
A=\left(\begin{array}{cc}
1 & 0 \\
-1 & 1
\end{array}\right) \quad \text { and } \quad A^{-1}=\left(\begin{array}{ll}
1 & 0 \\
1 & 1
\end{array}\right)
$$




\subsection{Predictions of the model}

We now successively give the predictions of the model for the up quarks, down quarks, charged leptons and neutrinos.

\subsubsection{Up-quark masses}

The up-quark masses correspond to the invariant $S=Q \bar{u} H_{u}$. The charge $Y_{F}\left(H_{u}\right)$ is fixed by the fact that the top quark mass appears unsuppressed in the superpotential,

$$
Y_{F}\left(H_{u}\right)=-\frac{10}{3} \quad \text { and } \quad Y_{F}\left(H_{d}^{\prime}\right)=\frac{10}{3}
$$

We also have

$$
X^{[u]} \equiv X\left(Q \bar{u} H_{u}\right)=0
$$

Therefore, the Yukawa matrix for the up quarks has the following structure

$$
Y^{[u]} \sim\left(\begin{array}{ccc}
\lambda^{6} & \lambda^{5} & \lambda^{3} \\
\lambda^{5} & \lambda^{4} & \lambda^{2} \\
\lambda^{3} & \lambda^{2} & 1
\end{array}\right)
$$

leading to the ratios

$$
\frac{m_{u}}{m_{t}} \sim \lambda^{6} \quad \frac{m_{c}}{m_{t}} \sim \lambda^{4}
$$

which are in agreement with phenomenology ${ }^{5}$.

\subsubsection{Down-quark masses}

Holomorphy of the terms containing the invariant $S=Q \bar{d} H_{d}$ forces the charge $Y_{F}\left(H_{d}\right)=n$ to be an integer, so that $H_{d}$ cannot be the vector partner of $H_{u}$. If $X^{[d]} \equiv X\left(Q \bar{d} H_{d}\right)$, the Yukawa matrix for the down quarks has the following structure in the absence of supersymmetric zeros (i.e. $X^{[d]} \leq 0$ is an integer and $\left.X^{[d]}+n+2 \leq 0\right)$

$$
Y^{[d]} \sim \lambda^{-2 X^{[d]}-2-n}\left(\begin{array}{ccc}
\lambda^{4} & \lambda^{3} & \lambda^{3} \\
\lambda^{3} & \lambda^{2} & \lambda^{2} \\
\lambda & 1 & 1
\end{array}\right)
$$

\footnotetext{
${ }^{5}$ Other models [11] 14] predict a smaller ratio $\frac{m_{u}}{m_{t}} \sim \lambda^{8}$, but both values are consistent with phenomenology
} 
leading to the interfamily hierarchy

$$
\frac{m_{d}}{m_{b}} \sim \lambda^{4} \quad \frac{m_{s}}{m_{b}} \sim \lambda^{2}
$$

Diagonalization of the Yukawa matrices $Y_{u}$ and $Y_{d}$ yields the correct Cabibbo structure for the CKM mixing matrix.

$$
U_{C K M} \sim\left(\begin{array}{ccc}
1 & \lambda & \lambda^{3} \\
\lambda & 1 & \lambda^{2} \\
\lambda^{3} & \lambda^{2} & 1
\end{array}\right)
$$

In this model, the intrafamily hierarchy between $m_{t}$ and $m_{b}$ is not predicted. We have

$$
\frac{m_{b}}{m_{t}} \sim \cot \beta \lambda^{-2 X^{[d]}-2-n}
$$

which has to be matched with the observed value

$$
\frac{m_{b}}{m_{t}} \sim \lambda^{3}
$$

\subsubsection{Charged lepton masses}

As the family charges of this model are unified into $S U(5)$ multiplets, it follows that the charge structure of the invariants $L \bar{e} H_{d}$ and $Q \bar{d} H_{d}$ are the same. Therefore,

$$
Y^{[e]} \sim \lambda^{-2 X^{[d]}-2-n}\left(\begin{array}{ccc}
\lambda^{4} & \lambda^{3} & \lambda^{1} \\
\lambda^{3} & \lambda^{2} & 1 \\
\lambda^{3} & \lambda^{2} & 1
\end{array}\right)
$$

leading to the ratios

$$
\frac{m_{e}}{m_{\tau}} \sim \lambda^{4} \quad \frac{m_{\mu}}{m_{\tau}} \sim \lambda^{2}
$$

A smaller ratio $\frac{m_{e}}{m_{\tau}} \sim \lambda^{5-6}$ would somehow be in better agreement with the measured masses, but $\lambda^{4}$ is not incompatible ${ }^{6}$. For the intrafamily hierarchy, the model predicts

$$
\frac{m_{b}}{m_{\tau}} \sim 1
$$

\footnotetext{
${ }^{6}$ The main problem with the $S U(5)$ unification of the charges is that the observed $m_{e} / m_{\mu}$ and $m_{d} / m_{s}$ ratios are different by a factor 10 . However, taking the ratio of the two light masses might not be a good "measure" of the agreement between the model and the data, because many order one coefficients are involved in this ratio. Rather, for $m_{d} \simeq 6 \mathrm{MeV}$, the ratios $m_{e} / m_{\tau}$ and $m_{d} / m_{b}$ are separated by a factor 4.5 only.

A mechanism to deal with this problem is also proposed in ref. [24]
} 
which indeed corresponds to what is observed. We take this fact as an experimental hint that the family symmetries are beyond $\mathrm{SU}(5)$.

\subsubsection{Neutrino masses}

The light neutrinos masses are obtained after seesaw. The Majorana mass term for the right-handed neutrinos is based on the invariant $M \bar{N} \bar{N}$. Similarly to the model of ref. [12, the flat direction analysis of the vacuum (see [25]) fixes the $X$-charge of $\bar{N}$

$$
X(\bar{N})=-\frac{3}{2}
$$

The right-handed neutrino Majorana mass matrix then contains a harmless supersymmetric zero in position 33 , and is given by

$$
M_{0} \sim M\left(\begin{array}{ccc}
\lambda^{10} & \lambda^{9} & \lambda^{5} \\
\lambda^{9} & \lambda^{8} & \lambda^{4} \\
\lambda^{5} & \lambda^{4} & 0
\end{array}\right)
$$

The order of magnitude of the massive right-handed neutrinos is therefore given by

$$
m_{\bar{N}_{1}} \sim M \lambda^{10} \quad m_{\bar{N}_{2}} \sim M \lambda^{8} \quad m_{\bar{N}_{1}} \sim M
$$

It is interesting to notice that the mass of the heaviest right-handed neutrino is not suppressed by any power of $\lambda$.

The right-handed neutrinos are coupled to the left-handed neutrino through the invariant $L \bar{N} H_{u}$. We notice that, with $X=\alpha+\beta V$, we have

$$
X^{[\nu]} \equiv X\left(L \bar{N} H_{u}\right)=X(L)+X(\bar{N})-X(Q)-X(\bar{u})=0
$$

for any values of $\alpha$ and $\beta$. As a result, the Dirac mass matrix is given by $\left(<H_{u}^{0}>=v_{u}\right)$

$$
m_{D} \sim v_{u}\left(\begin{array}{ccc}
\lambda^{6} & \lambda^{5} & \lambda \\
\lambda^{5} & \lambda^{4} & 1 \\
\lambda^{5} & \lambda^{4} & 1
\end{array}\right)
$$

The light neutrinos mass matrix obtained after see-saw is

$$
m_{\nu} \sim \frac{v_{u}^{2}}{M}\left(\begin{array}{ccc}
\lambda^{2} & \lambda & \lambda \\
\lambda & 1 & 1 \\
\lambda & 1 & 1
\end{array}\right)
$$


The measured value of $\Delta m_{\oplus}^{2}$ gives a phenomenological constraint on the scale $M$. We find

$$
M \sim 10^{16} \mathrm{GeV}
$$

which agrees very well with the string scale. The typical mass of the lightest right-handed neutrino is then $m_{\bar{N}_{1}} \sim 10^{9-10} \mathrm{GeV}$. For the purpose of leptogenesis, this value is somehow too small in the classical scheme of the decay of right-handed neutrinos [26]. But if leptogenesis occurs in a scheme where the Majorana neutrinos are only virtual, then the scale found here agrees with the thermodynamical conditions of the scenario [27.

We now turn back to the question of the significance of an approximate $P_{23}$ (or $\left.-P_{23}\right)$ symmetry. If this symmetry is somehow theoretically motivated, it will appear at the level of the superpotential, which will be symmetric (or antisymmetric) for the exchange of the lepton doublets $L_{2}$ and $L_{3}$. This will affect both the Dirac mass term and the charged leptons mass term. As a result, the muon mass will be suppressed by an extra power of $\lambda$, so that

$$
\frac{m_{\mu}}{m_{\tau}} \sim \lambda^{3}
$$

Moreover, the diagonalization $U_{-1}^{\dagger} Y^{[e]} V_{-1}$ and $U_{0}^{t} m_{\nu} U_{0}$ of the charged lepton and light neutrino masses $Y^{[e]}$ and $m_{\nu}$ yields a maximal mixing angle $\theta_{23}$ in both $U_{-1}$ and $U_{0}$. These cancel out in the MNS matrix $U_{M N S}=U_{-1}^{\dagger} U_{0}$, and the atmospheric mixing angle becomes small. Therefore, we conclude that the approximate $P_{23}$ symmetry is not phenomenologically acceptable in this model, if it is taken as a fundamental symmetry in the effective theory. However, an accidental approximate symmetry can still be viable.

\subsection{Anomaly cancellation}

The consistency of the model depends on anomaly cancellations. There are different possible anomalies that we will analyze in the following sequence: Standard Model gauge groups $G_{S M}$ anomalies $\left(G_{S M}\right.$ is $S U(3), S U(2)$ or $U(1)_{Y}$, mixed anomalies between $G_{S M}$ and $U(1)_{Y_{F}}, U(1)_{Y_{F}}^{3}$ anomaly, anomalies involving $U(1)_{X}$ and gravitational anomalies.

The anomaly coefficients involving only the Standard Model gauge group vanish over the chiral matter as well as over the vector-like pairs of Higgs, so $\left(G_{S M} G_{S M} G_{S M}\right)=0$.

The anomaly coefficients of the type $\left(Y_{F} G_{S M} G_{S M}\right)$ vanish over the three fermion families because $Y_{F}$ is traceless. They also vanish on the Higgs as 
these are vector-like with respect to $Y_{F}$. The anomaly coefficients $\left(Y_{F} Y_{F} G_{S M}\right)$ vanish automatically if $G_{S M}$ is either $S U(3)$ or $S U(2)$. The cancellation of the coefficients $\left(Y_{F} Y_{F} Y\right)$ is ensured over an $S U(5)$ multiplet because $Y_{F}$ lies outside $S U(5)$. Therefore,

$$
\left(Y_{F} Y_{F} Y\right)_{\mathbf{1 0}}=\left(Y_{F} Y_{F} Y\right)_{\overline{\mathbf{5}}}=0
$$

Finally, the right-handed neutrino has zero hypercharge and the vectorlike Higgs do not contribute. It is worth emphasizing that all the mixed anomaly coefficients involving $G_{S M}$ and $Y_{F}$ vanish over the representation 16 of $S O(10)$. Therefore, it is not necessary to extent the matter content of the model to the representation $\mathbf{2 7}$ of $E_{6}$ like in the model of ref. [12.

The anomaly coefficient $\left(Y_{F} Y_{F} Y_{F}\right)$ does not vanish over the three chiral families. We find

$$
\left(Y_{F} Y_{F} Y_{F}\right)_{\text {chiral }}=39+\frac{1}{9}
$$

We also expect some contribution from the $\theta$ sector. These anomalies need to be cancelled by some additional fields in the model that are singlet under the Standard Model gauge groups, but carry $X$ and $Y_{F}$ charges. The detailed structure of the additional matter fields necessary to cancel this anomaly is beyond the scope of the present paper, as this matter content does not have a phenomenological impact.

As mentioned, the family-independent symmetry $U(1)_{X}$ is necessarily anomalous. The anomalies linear in $X$ are compensated through the GreenSchwarz anomaly cancellation mechanism [22]. As a consequence, the value of the Weinberg angle at the cut-off $M$ is related to anomaly coefficients [23],

$$
\sin ^{2} \theta_{W}=\frac{C_{2}}{C_{1}+C_{2}}
$$

In our model, we have

$$
\begin{array}{r}
C_{1}=10 \alpha \\
C_{2}=C_{3}=6 \alpha
\end{array}
$$

so that the calculated value of $\sin ^{2} \theta_{W}$ is equal to the canonical value in $S U(5)$ theories,

$$
\sin ^{2} \theta_{W}=\frac{3}{8}
$$


which is a consequence of the fact that $U(1)_{X}$ lies outside $S U(5)$. The anomalies $\left(X Y_{F} Y_{F}\right)$ and $(X X X)$ are also compensated by the Green-Schwarz mechanism. All the anomaly coefficients which are quadratic in $X$ vanish. Obviously, we have $(X X S U(2))=(X X S U(3))=0$ and $\left(X X Y_{F}\right)=0$ (as usual, the vector-like Higgs do not contribute). Again, the fact that $U(1)_{X}$ does not lie into $S U(5)$ guarantees $(X X Y)=0$.

There are also gravitational anomalies. In particular, $C_{\text {grav }} \equiv(X T T) \neq$ 0 , where $T$ is the energy-momentum tensor, is also compensated by the Green-Schwarz mechanism. All the remaining possible anomaly coefficients involving $T$ vanish.

\section{Conclusions}

Neutrino physics is a privileged sector for the study of new physics beyond the Standard Model. The accumulated neutrino oscillation data over the last few years are precise enough to give serious hints on the structure of a possible unifying theory. They not only indicate the existence of neutrinos masses, the see-saw mechanism also provides a unique opportunity to glimpse some mysteries at scales as high as the unification scale.

In the spirit of a hierarchical unifying structure, we have found that a simple neutrino mass matrix is suggested by the present data on neutrino oscillations. It can accommodate two large and one small mixing angles without strong fine-tuning.

In the language of an effective theory with Abelian family symmetries, the lepton doublets charges derived from this matrix, when combined with the hierarchy and mixing structure observed in the quark sector, give a strong indication that the family symmetries lie beyond $S U(5)$. With the explicit construction of such a model, we see that this simple picture is indeed in a remarkable agreement with all the present data on quarks and leptons masses and mixings. Moreover, the model is by itself theoretically consistent and economic. It exhibits anomaly cancellation, and the correct energy scales for the string cut-off and for leptogenesis are obtained. 


\section{Acknowledgments}

We are thankful to Stuart Wick for helpful discussions throughout this work, which is supported by the United States Department of Energy under grant DE-FG02-97ER41029.

\section{Appendix: Mixing matrix and eigenvalues of $M_{\nu}^{(2)}$ for $D_{0} \sim O\left(\lambda^{2}\right)$ and $D_{1} \sim O\left(\lambda^{2}\right)$}

The formulas given below assume that the expansion in $\lambda$ is valid. For simplicity, we also neglect $\mathrm{CP}$ violating phases, so that all the matrix elements are real, and the mixing matrix is orthogonal. Then, for $D_{0} \sim O\left(\lambda^{2}\right)$ and $D_{1} \sim O\left(\lambda^{2}\right), M_{\nu}^{(2)}$ can be parameterized as

$$
M_{\nu}^{(2)}=m_{0}\left(\begin{array}{ccc}
a \lambda^{2} & d \lambda & f \lambda \\
d \lambda & b & e \\
f \lambda & e & c
\end{array}\right)
$$

with

$$
\begin{aligned}
b & =\gamma \sin ^{2} \theta+b_{1} \lambda+b_{2} \lambda^{2} \\
c & =\gamma \cos ^{2} \theta+c_{1} \lambda+c_{2} \lambda^{2} \\
e & =\gamma \sin \theta \cos \theta+e_{1} \lambda+e_{2} \lambda^{2} \\
d & =\beta \sin \theta+d_{1} \lambda+d_{2} \lambda^{2} \\
f & =\beta \cos \theta+f_{1} \lambda+f_{2} \lambda^{2}
\end{aligned}
$$

where all the coefficients $\theta, \gamma, \beta, b_{1}, \ldots$ are supposed to be of order $O(1)$.

The condition $D_{1} \equiv \delta_{1} \lambda^{2}$ gives the relation

$$
\cos ^{2} \theta b_{1}+\sin ^{2} \theta c_{1}-2 \sin \theta \cos \theta e_{1}=0
$$

and

$$
\delta_{1}=\delta_{1}^{\prime}+\gamma \delta_{2}^{\prime}
$$

with

$$
\begin{aligned}
& \delta_{1}^{\prime}=b_{1} c_{1}-e_{1}^{2} \\
& \delta_{2}^{\prime}=\cos ^{2} \theta b_{2}+\sin ^{2} \theta c_{2}-2 \sin \theta \cos \theta e_{2}
\end{aligned}
$$


It can then be verified that $D_{0} \equiv \delta_{0} \lambda^{2}$ with

$$
\delta_{0}=a \delta_{1}-\gamma \kappa^{2}-2 \beta \kappa \sigma-\beta^{2} \delta_{2}^{\prime}
$$

and

$$
\begin{aligned}
\kappa & =d_{1} \cos \theta-f_{1} \sin \theta \\
\sigma & =e_{1}-b_{1} \cot \theta
\end{aligned}
$$

The eigenvalues of $M_{\nu}^{(2)}$ are given by

$$
\mu_{0,1} \simeq m_{0} k_{0,1} \lambda^{2} \quad \mu_{2} \simeq m_{0} \gamma
$$

with

$$
k_{0,1}=\frac{\left(E+\delta_{1}\right) \pm \sqrt{\left(E+\delta_{1}\right)^{2}-4 \gamma \delta_{0}}}{2 \gamma}
$$

and $E=a \gamma-\beta^{2}$.

The mixing matrix is given by

$$
U \simeq\left(\begin{array}{ccc}
\cos \phi & \sin \phi & \frac{\beta}{\gamma} \lambda \\
-\sin \phi \cos \theta & \cos \phi \cos \theta & \sin \theta \\
\sin \phi \sin \theta & -\cos \phi \sin \theta & \cos \theta
\end{array}\right)
$$

with

$$
\tan \phi=\frac{a \gamma-k_{0} \gamma-\beta^{2}}{\gamma \kappa+\beta \sigma}=-\frac{\gamma \kappa+\beta \sigma}{a \gamma-k_{1} \gamma-\beta^{2}}
$$

Consistency for the value of $\tan \phi$ requires that

$$
\left(a \gamma-k_{0} \gamma-\beta^{2}\right)\left(a \gamma-k_{1} \gamma-\beta^{2}\right)+(\gamma \kappa+\beta \sigma)^{2}=0
$$

which indeed can be verified using the fact that $\sigma^{2}=-\delta_{1}^{\prime}$.

\section{References}

[1] T. Toshito for the SK Coll., Proceedings of the XXXVIth Rencontres de Moriond, hep-ex/0105023.

[2] The Super-Kamiokande Collaboration, Phys. Rev. Lett. 85, 3999 (2000). 
[3] J.N. Bahcall, M.C. Gonzalez-Garcia and C. Peña-Garay, JHEP 0204, 007 (2002).

[4] M. B. Smy for SK, Proceedings of the XXXVIth Rencontres de Moriond, hep-ex/0106064.

[5] SNO Collaboration, Phys. Rev. Lett. 87, 071301 (2001); SNO Collaboration, nucl-ex/0204008.

[6] SNO Collaboration, nucl-ex/0204009.

[7] The LSND collaboration, Phys. Rev. Lett. 77, 3082 (1996); The LSND collaboration, Phys. Rev. Lett. 81, 1774 (1998).

[8] M. Apollonio et al., Phys. Lett. B 338, 383 (1998); M. Apollonio et al., Phys. Lett. B 420, 397 (1998).

[9] S.F. King, hep-ph/0204360.

[10] Y. Grossman, Y. Nir and Y. Shadmi, JHEP 9810, 007 (1998); G. Altarelli and F. Feruglio, Phys. Rept. 320, 295 (1999); M.S. Berger and K. Siyeon, Phys. Rev. D 64, 053006 (2001).

[11] J.K. Elwood, N. Irges and P. Ramond, Phys. Rev. Lett. 81, 5064 (1998).

[12] N. Irges, S. Lavignac and P. Ramond, Phys. Rev. D 58, 035003 (1998).

[13] S. Lavignac, I. Masina, C.A. Savoy, Phys. Lett. B 520, 269 (2001).

[14] M.S. Berger and K. Siyeon, Phys. Rev. D 63, 057302 (2001).

[15] J. Sato and T. Yanagida, Phys. Lett. B 493, 356 (2000).

[16] F. Vissani, JHEP 9811, 025 (1998); F. Vissani, Phys. Lett. B 508, 79 (2001).

[17] A. Aranda, C.D. Carone and P. Meade, Phys. Rev. D 65, 013011 (2002).

[18] S.J. Huber and Q. Shafi, hep-ph/0205327.

[19] M. Bando and N. Maekawa, Prog. Theor. Phys. 106, 1255 (2001); N. Maekawa, Prog. Theor. Phys. 107, 597 (2002). 
[20] M. Gell-Mann, P. Ramond, and R. Slansky in Sanibel Talk, CALT-68709, Feb 1979 retropreprinted as hep-ph/9809459, and in Supergravity (North Holland, Amsterdam 1979). T. Yanagida, in Proceedings of the Workshop on Unified Theory and Baryon Number of the Universe, KEK, Japan, Feb 1979.

[21] C. Froggatt and H.B. Nielsen, Nucl. Phys. B 147, 277 (1979).

[22] M. Green and J. Schwarz, Phys. Lett. B 149, 117 (1984).

[23] L. Ibañez, Phys. Lett. B 303, 55 (1993).

[24] J. Ellis and M.K. Gaillard, Phys. Lett. B 88, 315 (1979); G. Altarelli, F. Feruglio and I. Masina, Phys. Lett. B 472, 382 (2000).

[25] P. Binétruy, N. Irges, S. Lavignac and P. Ramond, Phys. Lett. B 403, 38 (1997).

[26] M. Fukugita and T. Yanagida, Phys. Lett. B 174, 45 (1986).

[27] J.-M. Frère, F.-S. Ling, M. Tytgat, and V. Van Elewyck, Phys. Rev. D 60, 016005 (1999). 